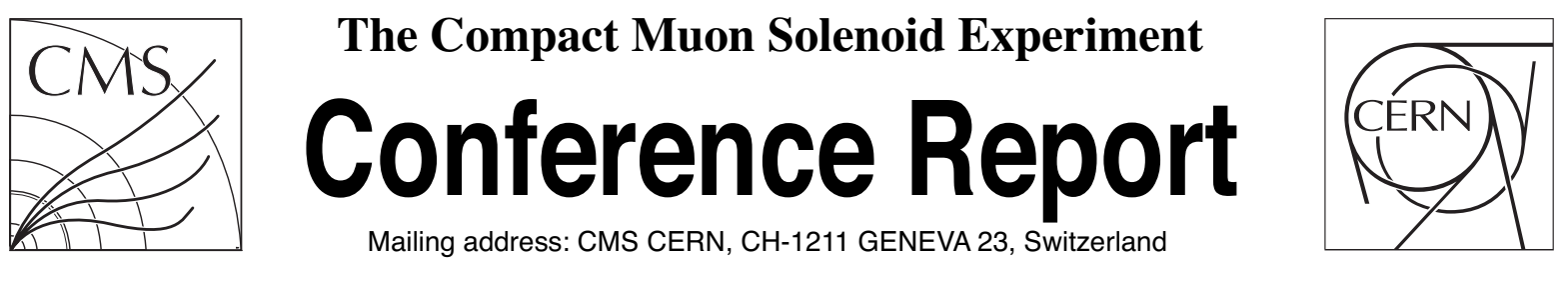

23 April 2020 (v7, 06 August 2021)

\title{
Front-End electronics for CMS iRPC detectors
}

\author{
Konstantin Shchablo for the CMS Collaboration
}

\begin{abstract}
A new generation of resistive plate chambers, capable of withstanding high particle fluxes (up to $2000 \mathrm{~Hz} \cdot \mathrm{cm}^{-2}$ ) and instrumented with precise timing readout electronics is proposed to equip two of the four high pseudorapidity stations of the CMS muon system. Double-gap RPC detectors, with each gap made of two $1.4 \mathrm{~mm}$ High Pressure Laminate electrodes and separated by a gas gap of the same thickness, are proposed. The new scheme reduces the amount of the avalanche charge produced by the passage of a charged particle through the detector. This improves the RPC rate capability by reducing the needed time to collect this charge. To keep the RPC efficiency high, a sensitive, low-noise and high time resolution front-end electronics is needed to cope with the lower charge signal of the new RPC. An ASIC called PETIROC that has all these characteristics has been selected to read out the stripes of new chambers. A thin $(0.6 \mathrm{~mm})$ printed circuit board, $160 \mathrm{~cm}$ long, equipped with pickup strips of $0.75 \mathrm{~cm}$ average pitch, will be inserted between the two new RPC's gaps. The strips will be read out from both ends, and the arrival time difference of the two ends will be used to determine the hit position along the strip. Results from the improved RPC equipped with the new readout system and exposed to muon beams in the high irradiation environment at the CERN GIF++ facility are presented for two versions of front-end electronics in this work.
\end{abstract}


PREPARED FOR SUBMISSION TO JINST

$\mathrm{XV}^{\mathrm{Th}}$ Workshop on Resistive Plate Chambers and Related Detectors RPC 2020

10-14 FEBruary 2020

Aula Magna "P.Gismondi", Via Della Ricerca Scientifica 1, 00133, Roma

\section{Front-End electronics for CMS iRPC detectors}

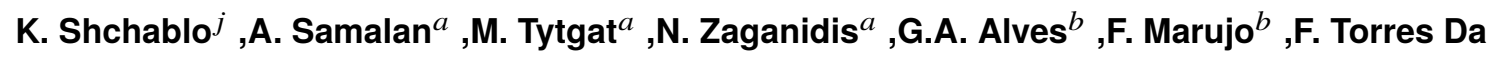
Silva De Araujo ${ }^{c}$, ,E.M. Da Costa $^{c}$,D. De Jesus Damiao ${ }^{c}$, H. Nogima ${ }^{c}$,A. Santoro ${ }^{c}$,S. Fonseca De Souza $^{c}$, A. Aleksandrov ${ }^{d}$, R. Hadjiiska $^{d}$, ,P. laydjiev $^{d}$, M. Rodozov $^{d}, \mathbf{M}$.

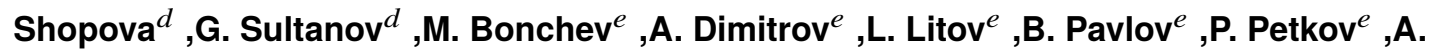
Petrov $^{e}$,S.J. Qian ${ }^{f}$,C. Bernal ${ }^{g}$,A. Cabrera ${ }^{g}$, ,J. Fraga $^{g}$,A. Sarkar ${ }^{g}$,S. Elsayed ${ }^{h}$,Y. Assran $^{h h, h h h}$,M. El Sawy $h h, h h h h$,M.A. Mahmoud ${ }^{i}$, ,Y Mohammed $^{i}$,X. Chen $^{j}$,C. Combaret $^{j}$ ,M. Gouzevitch ${ }^{j}$,G. Grenier ${ }^{j}$, ,I. Laktineh ${ }^{j}$,L. Mirabito ${ }^{j}$, ,I. Bagaturia $^{k}$,D. Lomidze ${ }^{k}$,I.

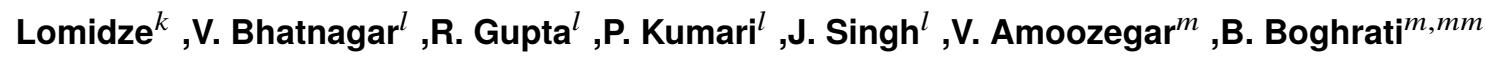
,M. Ebraimi ${ }^{m}$, R. Ghasemi ${ }^{m}$, M. Mohammadi Najafabadi ${ }^{m}$, E. Zareian ${ }^{m}$, M. Abbrescia ${ }^{n}, \mathbf{R}$.

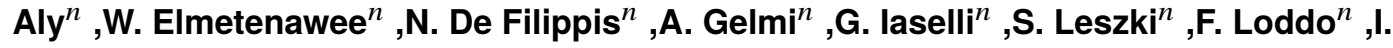
Margjeka $^{n}$, G. Pugliese $^{n}$,D. Ramos ${ }^{n}$,L. Benussi ${ }^{o}$, ,S. Bianco ${ }^{o}$, ,D. Piccolo ${ }^{o}$,S. Buontempo ${ }^{p}$ ,A. Di Crescenzo ${ }^{p}{ }^{\text {,F. Fienga }}{ }^{p}$,G. De Lellis ${ }^{p}$,L. Lista ${ }^{p}$,S. Meola ${ }^{p}$, P. Paolucci ${ }^{p}$,A. Braghieri ${ }^{q}$ ,P. Salvini ${ }^{q}$,P. Montagna ${ }^{q q}$,C. Riccardi ${ }^{q q}$,P. Vitulo ${ }^{q q}$,B. Francois ${ }^{r}$,T.J. Kim ${ }^{r}$,J. Park ${ }^{r}$,S.Y.

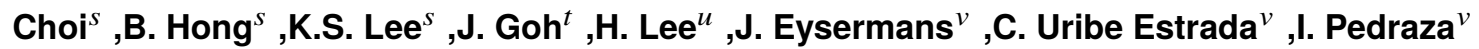
,H. Castilla-Valdez ${ }^{w}$, A. Sanchez-Hernandez ${ }^{w}$, ,C.A. Mondragon Herrera ${ }^{w}$,D.A. Perez

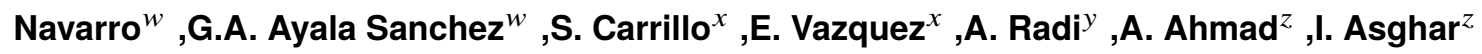
,H. Hoorani ${ }^{z}$,S. Muhammad ${ }^{z}$, M.A. Shah ${ }^{z}$,I. Crotty ${ }^{a a}$ on behalf of the CMS Collaboration

${ }^{a}$ Ghent University, Dept. of Physics and Astronomy, Proeftuinstraat 86, B-9000 Ghent, Belgium

${ }^{b}$ Centro Brasileiro Pesquisas Fisicas, R. Dr. Xavier Sigaud, 150 - Urca, Rio de Janeiro - RJ, 22290-180, Brazil

${ }^{c}$ Dep. de Fisica Nuclear e Altas Energias, Instituto de Fisica, Universidade do Estado do Rio de Janeiro, Rua Sao Francisco Xavier, 524, BR - Rio de Janeiro 20559-900, RJ, Brazil

${ }^{d}$ Bulgarian Academy of Sciences, Inst. for Nucl. Res. and Nucl. Energy, Tzarigradsko shaussee Boulevard 72, BG-1784 Sofia, Bulgaria.

${ }^{e}$ Faculty of Physics, University of Sofia,5 James Bourchier Boulevard, BG-1164 Sofia, Bulgaria.

${ }^{f}$ School of Physics, Peking University, Beijing 100871, China.

${ }^{g}$ Universidad de Los Andes, Apartado Aereo 4976, Carrera 1E, no. 18A 10, CO-Bogota, Colombia.

${ }^{h}$ Egyptian Network for High Energy Physics, Academy of Scientific Research and Technology, 101 Kasr El-Einy St. Cairo Egypt.

${ }^{h h}$ The British University in Egypt (BUE), Elsherouk City, Suez Desert Road, Cairo 11837- P.O. Box 43, Egypt.

${ }^{h h h h}$ Department of Physics, Faculty of Science, Beni-Suef University, Beni-Suef, Egypt

${ }^{i}$ Center for High Energy Physics, Faculty of Science, Fayoum University, 63514 El-Fayoum, Egypt. 
${ }^{j}$ Univ Lyon, Univ Claude Bernard Lyon 1, CNRS/IN2P3, IP2I Lyon, UMR 5822, F-69622, Villeurbanne, France.

${ }^{k}$ Georgian Technical University, 77 Kostava Str., Tbilisi 0175, Georgia

${ }^{l}$ Department of Physics, Panjab University, Chandigarh 160 014, India

${ }^{m}$ School of Particles and Accelerators, Institute for Research in Fundamental Sciences (IPM), P.O. Box 19395-5531, Tehran, Iran

${ }^{m m}$ School of Engineering, Damghan University, Damghan, 3671641167, Iran

${ }^{n}$ INFN, Sezione di Bari, Via Orabona 4, IT-70126 Bari, Italy.

${ }^{n n}$ ENEA, Frascati, Frascati (RM), I-00044, Italy

o INFN, Laboratori Nazionali di Frascati (LNF), Via Enrico Fermi 40, IT-00044 Frascati, Italy.

PINFN, Sezione di Napoli, Complesso Univ. Monte S. Angelo, Via Cintia, IT-80126 Napoli, Italy.

${ }^{q}$ INFN, Sezione di Pavia, Via Bassi 6, IT-Pavia, Italy.

${ }^{q q}$ INFN, Sezione di Pavia and University of Pavia, Via Bassi 6, IT-Pavia, Italy.

${ }^{r}$ Hanyang University, 222 Wangsimni-ro, Sageun-dong, Seongdong-gu, Seoul, Republic of Korea.

${ }^{s}$ Korea University, Department of Physics, 145 Anam-ro, Seongbuk-gu, Seoul 02841, Republic of Korea.

${ }^{t}$ Kyung Hee University, 26 Kyungheedae-ro, Hoegi-dong, Dongdaemun-gu, Seoul, Republic of Korea

"Sungkyunkwan University, 2066 Seobu-ro, Jangan-gu, Suwon, Gyeonggi-do 16419, Seoul, Republic of Korea

${ }^{v}$ Benemerita Universidad Autonoma de Puebla, Puebla, Mexico.

${ }^{w}$ Cinvestav, Av. Instituto Politécnico Nacional No. 2508, Colonia San Pedro Zacatenco, CP 07360, Ciudad de Mexico D.F., Mexico.

${ }^{x}$ Universidad Iberoamericana, Mexico City, Mexico.

${ }^{y}$ Sultan Qaboos University, Al Khoudh,Muscat 123, Oman.

${ }^{z}$ National Centre for Physics, Quaid-i-Azam University, Islamabad, Pakistan.

${ }^{a a}$ Dept. of Physics, Wisconsin University, Madison, WI 53706, United States.

E-mail: shchablo@ipnl.in2p3.fr

ABSTRACT: A new generation of resistive plate chambers, capable of withstanding high particle fluxes (up to $2000 \mathrm{~Hz} \cdot \mathrm{cm}^{-2}$ ) and instrumented with precise timing readout electronics is proposed to equip two of the four high pseudorapidity stations of the CMS muon system. Double-gap RPC detectors, with each gap made of two $1.4 \mathrm{~mm}$ High Pressure Laminate electrodes and separated by a gas gap of the same thickness, are proposed. The new layout reduces the amount of the avalanche charge produced by the passage of a charged particle through the detector. This improves the RPC rate capability by reducing the needed time to collect this charge. To keep the RPC efficiency high, a sensitive, low-noise and high time resolution front-end electronics is needed to cope with the lower charge signal of the new RPC. An ASIC called PETIROC that has all these characteristics has been selected to read out the strips of new chambers. Thin $(0.6 \mathrm{~mm})$ printed circuit board, $160 \mathrm{~cm}$ long, equipped with pickup strips of $0.75 \mathrm{~cm}$ average pitch, will be inserted between the two new RPC's gaps. The strips will be read out from both ends, and the arrival time difference of the two ends will be used to determine the hit position along the strip. Results from the improved RPC equipped with the new readout system and exposed to cosmic muons in the high irradiation environment at CERN GIF++ facility are presented in this work.

KeYwords: Gaseous detectors, Resistive Plate Chambers

\section{ArXIV EPRINT: XXXX.XXXXX}




\section{Contents}

1 Introduction 1

2 Front-End Board 2

3 Readout Algorithm 3

4 Performance of the iRPC with the new FEB 5

5 Conclusion and Plans $\quad 6$

\section{Introduction}

The improved RPC (iRPC) for the CMS experiment at the LHC is based on double-gap Resistive Plate Chambers (RPC) detectors with each gap made of two $1.4 \mathrm{~mm}$ High Pressure Laminate (HPL) electrodes and separated by a gas gap of the same thickness [2]. To read out the iRPC, a Front-End Board (FEB) is proposed. The prototype's layout with a new pickup strip panel is inserted between the two gaps shown in Figure 1.

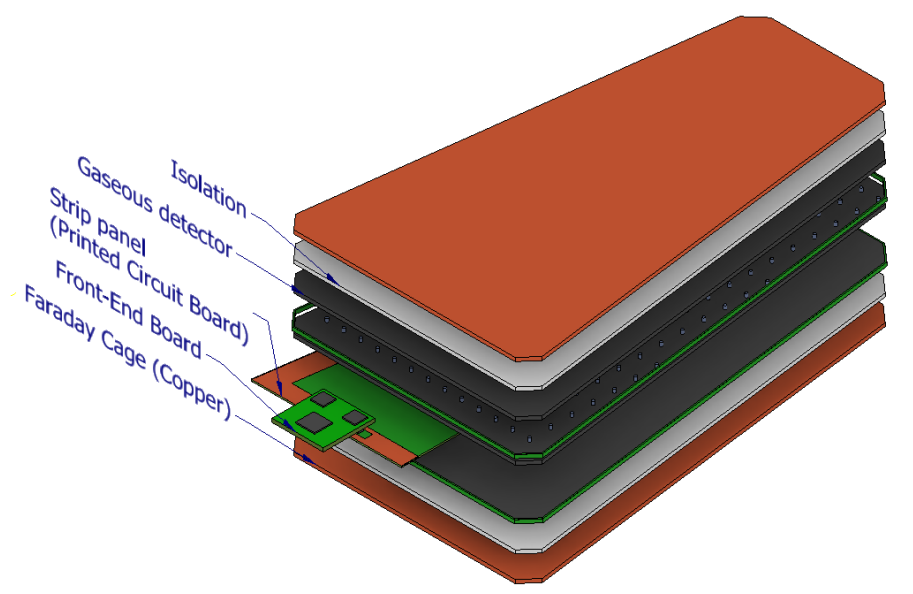

Figure 1: Schematic view of the layout of improved Resistive Plate Chamber.

The new FEB aims at keeping the iRPC efficiency as high as the current CMS RPC by using a sensitive, low-noise electronics and to increase the RPC spatial resolution thanks to high time resolution components. It will also sustain a much higher rate (up to $2000 \mathrm{~Hz} \cdot \mathrm{cm}^{-2}$ ) [1]. The PETIROC [3], an ASIC that has all of these characteristics, is proposed to perform the readout, in association with a high-resolution delay-line Time Digital Converter (TDC), implemented on a Field-Programmable Gate Array (FPGA) to digitize the signal collected by the strips. The new readout panel is made of a thin $(0.6 \mathrm{~mm})$ Printed Circuit Board (PCB) where each strip is read out 
on both ends. Thanks to the excellent time resolution of the electronics $(20-30 \mathrm{ps}[11])$, this $2 \mathrm{D}$ readout scheme allows one to use the arrival time difference of the two strip ends to determine the position along the strip with good resolution (about $200 \mathrm{ps}$ or $1.7 \mathrm{~cm}$ [9]). The FEB proposed for iRPC was tested on iRPC, exposed to cosmic muons in a high irradiation environment at the CERN GIF++ facility [5].

\section{Front-End Board}

In the FEB prototype, two principal components are employed: the Front-End (FE) electronics, using one or more PETIROC [3] ASICs, and an FPGA. Only the analog part of the PETIROC ASIC (Fig. 2) is exploited in the FEB. This includes a low-jitter and high bandwidth preamplifier followed by a precise discriminator that allows to operate at a charge threshold of $30 \mathrm{fC}$.

The output of the ASIC's discriminators is sent to the FPGA on which a precise delay-line TDC [6] is implemented. In addition to hosting the TDC, the FPGA ensures the communication between the FE and Back-End (BE) electronics. Indeed, by using FPGA, the received ASIC configuration and fast command from the $\mathrm{BE}$ are applied to the FE part, and collected data are sent back to the BE.
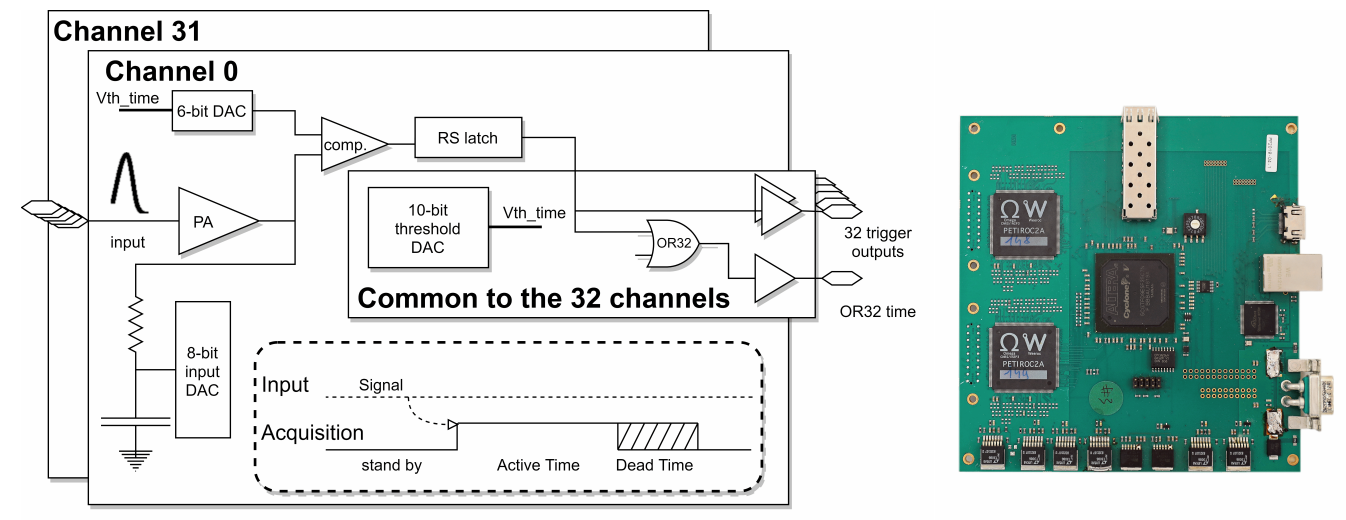

Figure 2: Left: Logical scheme of the analog part of PETIROC. Right: Pictures of the FEB.

The analog part of PETIROC includes a common 10-bit Digital to Analog Converter (DAC), which is used to ensure the adjustment of a global triggering level that can cover the whole dynamic range of analog signals for all channels. Furthermore, an individual 6-bit DAC is used in each channel to achieve a similar response for each specific channel to a given charge.

To reduce the observed retriggering behaviour of the channels of PETIROC ASIC when operated at threshold $(<160 \mathrm{fC})$, the ASIC must be set to operate in latch mode. In this mode, when the signal produced by a charged particle crossing the RPC passes the threshold of the ASIC's channel connected to the closest of the two strip's ends, the discriminator of this channel is neutralized for a period of $30 \mathrm{~ns}$. This period is the maximum time needed for the signal to reach the ASIC's channel that is connected to the other end of the fired strip. After this period, all channels of the ASIC are held on $\operatorname{reset}^{1}$ for a time interval whose length is chosen to stabilize the ASIC. For the

${ }^{1}$ The reset mode is global and involves all the ASIC PETIROC channels. 
different FEB prototypes developed so far, 10 ns was the lowest value that was possible to obtain. The structure of the readout and its timing diagram of the reset signal is illustrated in figure 2 .

To read out the iRPC, new FEB prototype has been developed and tested. The FEB, was equipped with two PETRIOC2B and an Altera Cyclone V FPGA. It was used to read out the strip panel. The strip panel hosts 48 strips and has a connector in which two ends of all the strips are electrically connected on the large side of the trapezoidal PCB (Fig. 3).

For the strip panel, the width of the pickup strips and their distance with respect to the reference grounding plane were optimized to have the same impedance all along the strip.

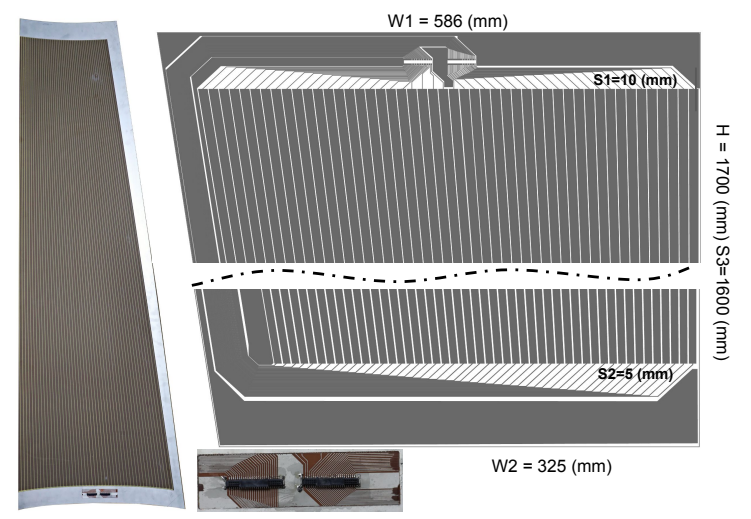

Figure 3: The strip panels with 48 strips.

Figure 3 shows the layout and key dimensions of the PCB strip panel with return lines that was tested. PCB was made using FR4 material. The dimensions given are for the panel widths (W1 and W2) of the High Radius (HR) and Low Radius (LR), the panel length (H), the strip pitch at the HR and LR sides (S1 and S2) and the average length of the strip (S3).

\section{Readout Algorithm}

Each end of a strip is connected to one PETIROC channel. A particle crossing the detector at a given position along the strip (Y) generates a signal induced on a pickup strip and split into two equal signals, which propagate respectively to each end of the strip. When the amplitude of the signal passes the threshold of the associated channel, the PETIROC generates an output signal to a TDC channel associated to the fired channel. In this way, the measurement of difference between the two signal arrival times allows the determination of the $Y$ position of the particle hit along the strip.

To study the iRPC detector's performance, we used an external trigger based on the coincident signals from three scintillation counters covering approximately $23 \mathrm{~cm}$ in the center of the chamber and corresponding to strips numbered $25-40$ of the chamber. This method allows us to study the efficiency of our detector to the cosmic muons in the corresponding area. The coincidence signals are also sent to one of the FPGA TDC channels to determine the arrival time.

Figure 4 shows the profile of the difference between the trigger time and the signal arrival time to the channels associated with the same strip, at the high radius and low radius ends, respectively. 
The width of these time profiles includes the impact on the position difference due to the trigger acceptance surface, the jitter of trigger time and the single strips' readout offsets.
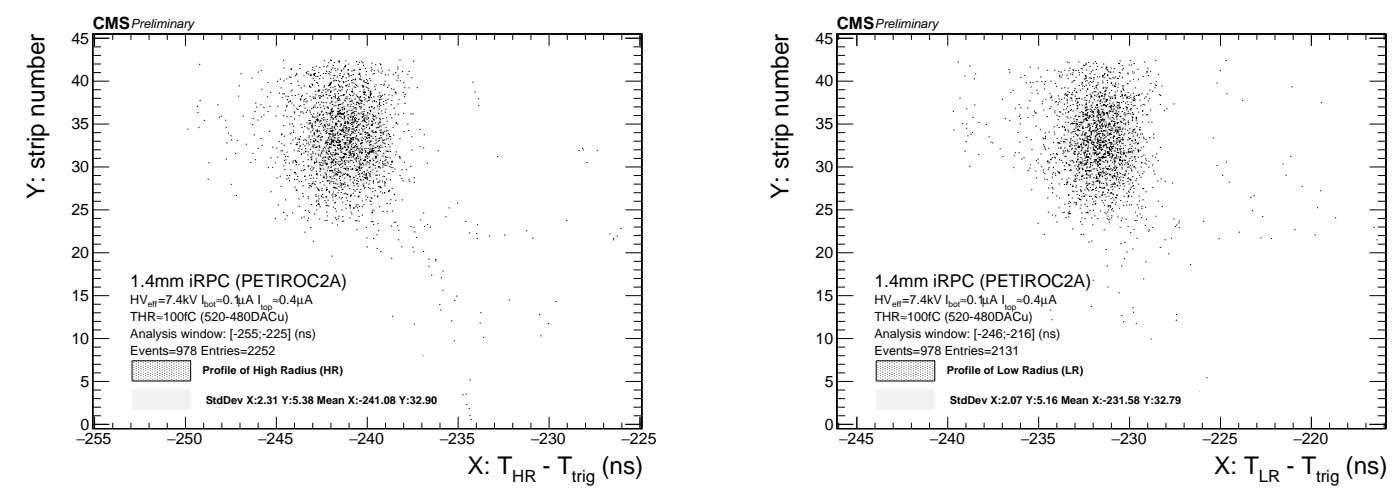

Figure 4: The time difference between readout strips arrival time and the trigger time (at plateau of efficiency). Left: FE channels connected to the strip ends at high radius. Right: FE channels connected to the strip ends at low radius.

The particle crossing position is reconstructed with a clustering procedure (average multiplicity is around 3). The clusters are built from fired channels (hits) from the two PCB sides separately.

The clusters of the two sides, which belong to the same group of strips and are compatible with the trigger time, are associated if the position estimated by using the difference of the average time of the two clusters is compatible with a position located on the detector.

In the opposite case these one-side clusters are tagged as such and are used to control the loss of efficiency due to the signal propagation. The clustering algorithm provides the profile of the triggered events shown in Figure 5.
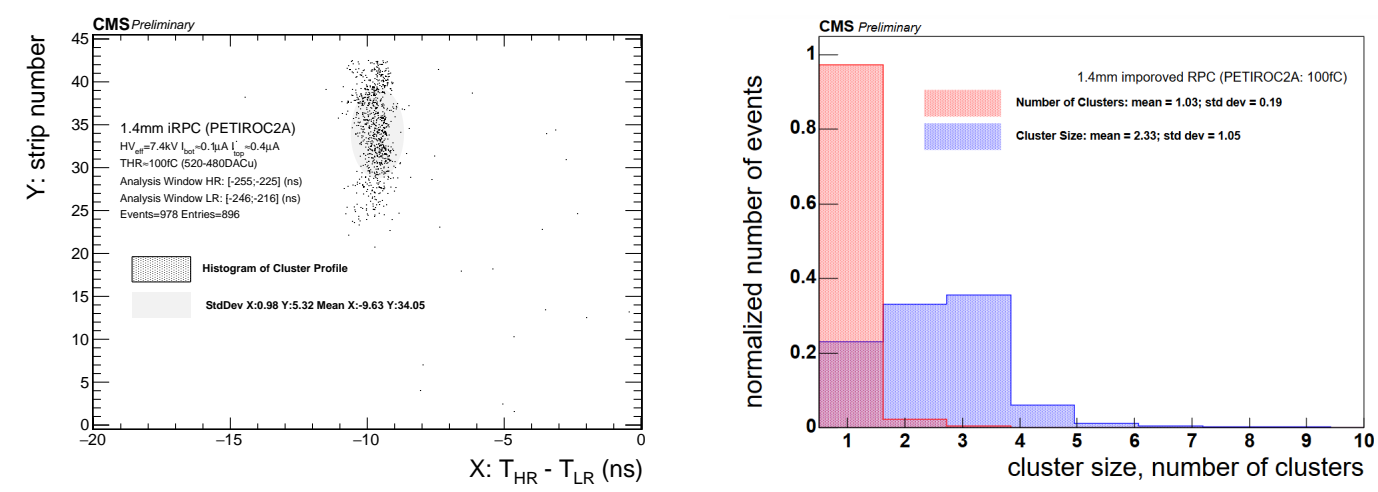

Figure 5: Left: The strip profile of the triggered events of the iRPCs as a function of the time difference. Right: Distributions of the cluster size and the number of clusters for the collected muon events.

The profile position and its spread of about $2 \mathrm{~ns}$ in the $\mathrm{X}$ direction are in agreement with those of the trigger system. Important outputs of the clustering algorithm are the number of clusters per muon and their multiplicity. As expected, in most cases only one cluster is found per event trigger (Fig. 5). 


\section{Performance of the iRPC with the new FEB}

To estimate the efficiency of the iRPC readout with the new FEB a system made of scintillatorPMTs setup was used as an external trigger. The cosmic trigger system was placed inside the GIF++ bunker protected from the gamma source by lead bricks. This trigger allows the study of the iRPC effciency in different gamma irradiation environments.

The efficiency of iRPC for cosmic muons is estimated as the number of triggers having at least one hit in the time interval associated to the trigger time arrival. For each trigger, the signal associated with the passage of the muon counter is incremented if a hit is found in each of the two time windows mentioned above. However, gammas background associated hits can occur in these two time windows and fake our muon counting. Thus, to estimate the true muon efficiency (Eq. 4.1), we have to subtract this background contribution assuming no correlation between background hits and muon hits.

$$
\varepsilon=\frac{\frac{N}{N_{\text {trig }}}-\frac{N_{\text {bkg }}}{N_{\text {trig }}}}{1-\frac{N_{b k g}}{N_{\text {trig }}}}
$$

Here, $\varepsilon, N, N_{\text {trig }}, N_{b k g}$ are the estimated muon efficiency, the number of the detected events with at least one hit, the number of muon triggers, and the number of gamma background events expected in the trigger time interval, respectively.

In principle, the GIF++ source produced a uniform irradiation all over the chamber. However, the presence of the lead shield resulted in different irradiation fluxes over different regions of the iRPC. This variation allows us to study the impact of the electronics dead time on the iRPC efficiency under a high flux counting rate (up to $2 \mathrm{kHz} \cdot \mathrm{cm}^{-2}$ ) while minimizing the drop in efficiency due to the detector itself.

Indeed the dead time of the electronics is sensitive to the average flux all over the iRPC, even if the detector efficiency is measured in the shielded region where the flux is about ten times lower.
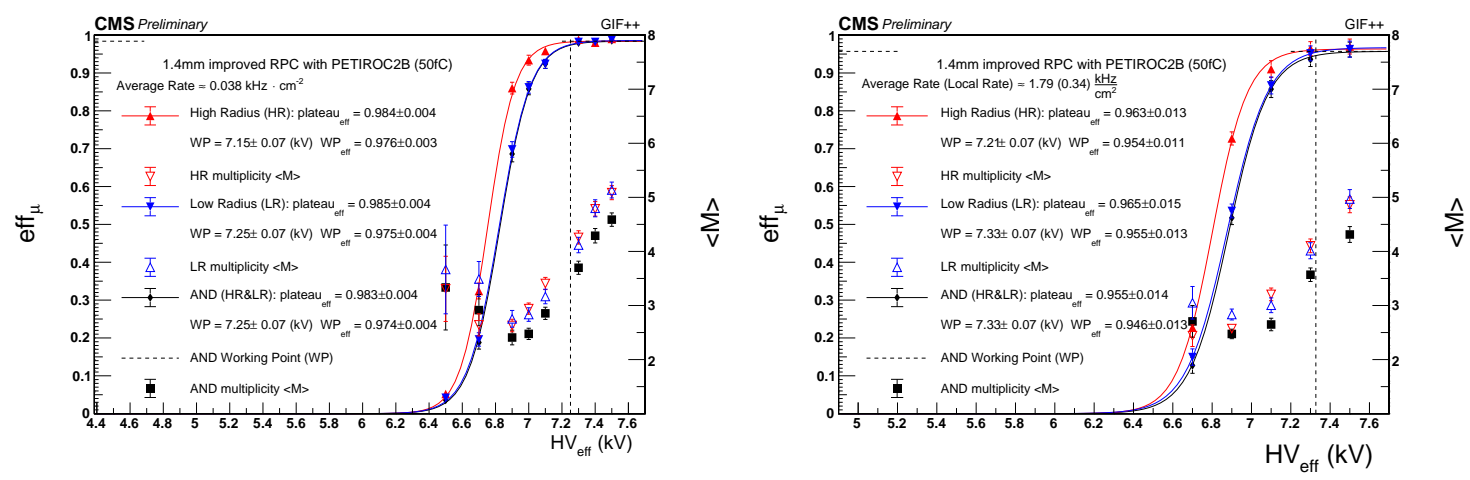

Figure 6: Efficiency versus effective high voltage without (left) and with (right) background rates. The number of hits per event is reported too.

Figure 6 shows the measured iRPC efficiency with a low irradiation (left) and a high one about $2 \mathrm{kHz} \cdot \mathrm{cm}^{-2}$ (right) versus the effective High Voltage (HV) [10] applied on each of the two gaps. 
The efficiency of the iRPC with a signal detected on the HR side, on the LR side, and on both of them are shown for the two irradiation scenarios.

It is important to note that the efficiency was measured in a position close to the high radius (HR) side of the chamber. This corresponds to the worst situation due to the signal loss through propagation along the strip. The signal in this case has to cross more than $3.6 \mathrm{~m}$ to reach the channels associated to the low radius (LR) side of the detector. This explains the relative difference in efficiencies observed between the high and low radius sides. This result was obtained with a PCB made of FR4 material with a dielectrics constant about 4.3. This effect is expected to be reduced with a EM888 material with typical dielectrics values 3.7, which is proposed to equip the future strip panels.

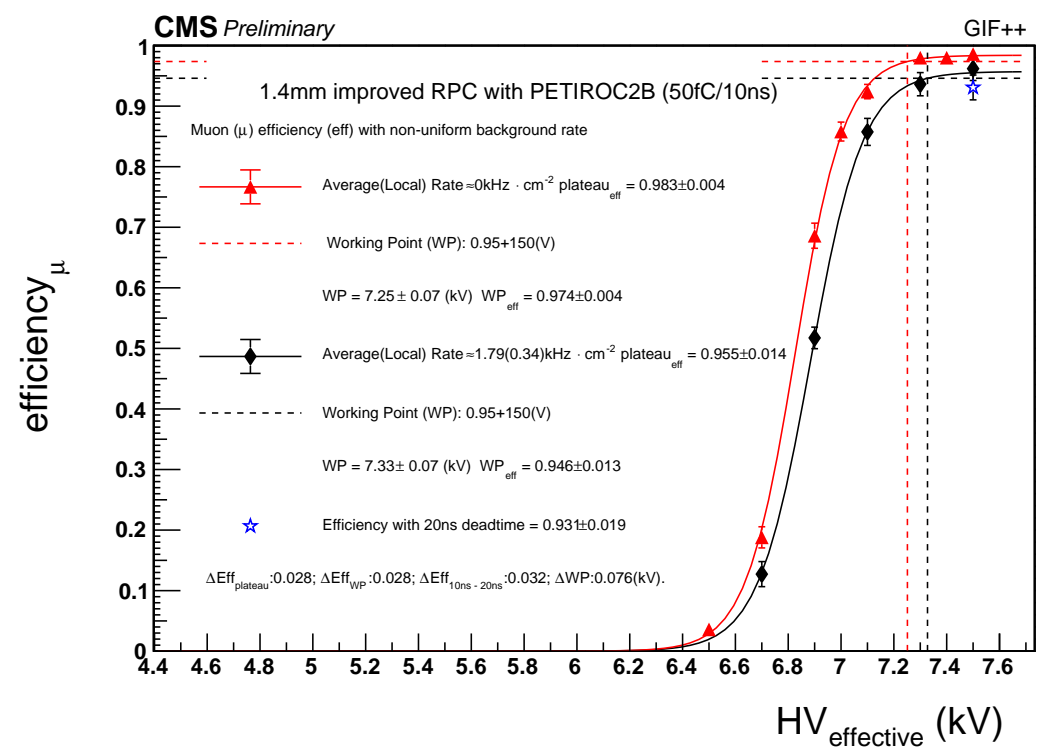

Figure 7: Study of the iRPC efficiency loss due to the FEB's dead time in presence of different gamma irradiation fluxes.

Figure 7 shows a drop in efficiency at $1.8 \mathrm{kHz} \cdot \mathrm{cm}^{-2}$ of about $2.8 \%$. As a cross-check, the efficiency was also measured under the same conditions but with a dead time of $20 \mathrm{~ns}$ which results in a $3 \%$ efficiency drop with respect to the one obtained at the same HV point with $10 \mathrm{~ns}$.

\section{Conclusion and Plans}

The version of the FEB based on PETIROC 2B for iRPC was developed and tested with $50 \mathrm{fC}$ threshold, without and with high gamma irradiation. Also, the drop in efficiency due to dead time was studied and shown to be around $2.8 \%$ at $1.8 \mathrm{kHz} \cdot \mathrm{cm}^{-2}$. To reduce the impact of this dead time on the iRPC at this very high flux, a new version of PETIROC with individual channel reset is being developed. The future PETIROC $2 \mathrm{C}$ should minimize the loss of efficiency. In addition, new material (EM888) will be used to build the dielectrics of the strip panels. This should minimize the loss of efficiency arising from the signal attenuation when propagating along the strip. 


\section{References}

[1] Collaboration, CMS, “The Phase-2 Upgrade of the CMS Muon Detectors," CERN-LHCC-2017-012, No. CERN-LHCC-2017-012. CMS-TDR-016,(Sep 2017).

[2] Lee, K. et al.,"Study of Thin Double-Gap RPCs for the CMS Muon System," Journal of the Korean Physical Society, Vol. 73, 1080-1087, (10 2018).

[3] J. Fleury,"Callier," de La Taille, Vol. Seguin, No. Thienpont, Dulucq, (Ahmad).

[4] Petiroc, a new front-end ASIC for time of flight application,"2013 IEEE Nuclear Science Symposium and Medical Imaging Conference," , Vol. , No. 1-5, 2013

[5] R. Guida,"GIF++: The new CERN Irradiation Facility to test large-area detectors for HL-LHC," 2015 IEEE Nuclear Science Symposium and Medical Imaging Conference, Vol. , No. , 1-4,(2015).

[6] J. Song, "A high-resolution time-to-digital converter implemented in field-programmable-gate-arrays," IEEE Transactions on Nuclear Science, Vol. 53, No. 1, 236-241, (2006).

[7] Chen et al., "Improved Tapped-Delay-Line Time-to-Digital Converter with Time-over-Threshold measurement for a new generation of Resistive Plate Chamber detectors," , 141,(05 2019).

[8] M. Gouzevitch et al.,"High rate, fast timing Glass RPC for the high $\eta$ CMS muon detectors," Nuclear Instruments and Methods in Physics Research Section A: Accelerators, Spectrometers, Detectors and Associated Equipment, Vol. 845, 226 - 227,(2017).

[9] K. Shchablo et al.,"Performance of the CMS RPC upgrade using 2D fast timing readout system," Nuclear Instruments and Methods in Physics Research Section A: Accelerators, Spectrometers, Detectors and Associated Equipment, Vol. 958, 162139,(2020).

[10] P. Pierluigi et al., "CMS Resistive Plate Chamber overview, from the present system to the upgrade phase I," Journal of Instrumentation, Vol. 8, P04005,(04 2013).

[11] Lagarde F. et al. High rate, fast timing Glass RPC for the high $\eta$ CMS muon detectors. Journal Of Instrumentation. 11, C09006-C0900 (2016) 